\title{
Globales Lernen in der Schule - Partizipation im Spannungsfeld von Inklusion und Exklusion
}

\begin{abstract}
Zusammenfassung
Partizipation ist ein weithin geforderter pädagogischer Anspruch, der derzeit insbesondere im Rahmen von inklusiver Beschulung, aber auch schon länger im Globalen Lernen bzw. in einer Bildung für nachhaltige Entwicklung formuliert wird. Vor diesem Hintergrund befasst sich der Beitrag mit der Frage, wie der Anspruch Globalen Lernens bzw. einer Bildung für nachhaltige Entwicklung, ein gutes Leben für alle anzustreben und hierfür die Beteiligung abstrakter Abwesender mitzudenken, für die pädagogische Praxis in der Schule theoretisch gefasst werden kann. Es wird argumentiert, dass Globales Lernen als Forderung nach partizipationsorientiertem Denken und Handeln auf der Ebene der Alltagspraktiken in einem Wechselspiel situativer Inklusion und Exklusion stattfindet - ein Spannungsfeld, dessen Komplexität sich durch die Berücksichtigung weltgesellschaftlicher Eingebundenheit noch einmal erhöht. Eingebettet in ein inklusiv angelegtes normatives Rahmenkonzept kann die Auseinandersetzung mit diesem Spannungsfeld dazu anregen, Unterrichtspraktiken und Lehr-Lernsettings bezogen auf damit einhergehende Differenzkonstruktionen und Partizipationsmöglichkeiten reflexiv zu befragen.
\end{abstract}

Schlüsselworte: Schule, Partizipation, Inklusion, Exklusion

\footnotetext{
Abstract

Participation is a demanded pedagogical claim, which is currently expressed especially in the context of inclusive schooling, but longer already in Global Learning or Education for Sustainable Development. Against this background, the paper deals with the question of how the claim of global learning or education for sustainable development that aims for a good life for all and that the participation of ,abstract absentees" could be theoretically formulated for pedagogical practice in schools. It is argued that global learning as a demand for participative action at the level of everyday practices takes place in an interplay of situational inclusion and exclusion - a field of tension whose complexity is further increased by the consideration of global social integration. Embedded in an inclusive normative conceptual framework, the discussion in this field of tension
}

can stimulate the reflexive questioning of teaching practices and teaching-learning settings with regard to the associated constructions of difference and possibilities of participation.

Keywords: participation, school, inclusion, exclusion

\section{Partizipation als aktive Teilhabe: Anforderung aus weltgesellschaftlicher Perspektive}

Im Globalen Lernen und einer Bildung für nachhaltige Entwicklung wird die Ermöglichung einer aktiven Teilhabe sowie des Empowerments als Grundlage der Mitgestaltung in einer Weltgesellschaft angestrebt (z. B. BMZ/KMK, 2015; de Haan, 2010; Scheunpflug, 2017; Stoltenberg, 2007). Kinder und Jugendliche werden in diesem Rahmen vor allem als Gestalter/ -innen ihrer Zukunft adressiert, was als Prozess nur unter Beteiligung aller, und somit partizipativ und inklusiv gedacht werden kann (vgl. auch SDG 4, UN, 2015). Partizipation ist damit zugleich Voraussetzung und Anliegen entsprechender Bildungskonzepte und ihrer methodisch-didaktischen Implikation. Partizipation und Inklusion als Kinder- und Menschenrecht (vgl. z. B. Reitz, 2015) wird im Globalen Lernen zumeist als soziale und politische Partizipation formuliert. Damit wird auf einen gesellschaftlichen Gestaltungsauftrag (vgl. Böhme, 2019) einerseits sowie die Aufgabe diesen zu ermöglichen, andererseits, verwiesen. Im Feld des politischen Lernens und der Demokratiebildung wird das Erleben und Gestalten von Mitbestimmung vor allem als Einübung in die Entwicklung eines mündigen Bürgers/einer mündigen Bürgerin in einer demokratischen Gesellschaft verstanden (zur politischen Partizipation, z. B. Erdsiek-Rave \& John-Ohnesorg, 2015; Widmaier \& Overwien, 2013). Die in den unterschiedlichen Bildungskonzepten formulierte Zielsetzung partizipativer Bildung basiert auf der grundlegenden Kritik an einer Benachteiligung aufgrund sozialer Zugehörigkeit.

Ausgangspunkt der Zielformulierung Partizipation bildet zumeist eine als nicht hinreichend identifizierte Beteiligung, die kompensiert werden muss. Diese Forderung ist gerade vor dem Hintergrund heterogener Lebenslagen und damit 
verbundener unterschiedlicher Zugänge zu formalen Räumen der Teilhabe und den damit verbundenen Partizipationsmöglichkeiten kritisch zu befragen. Im Vordergrund dieses Beitrags steht das Interesse daran, wie ein partizipativer und inklusiver Bildungsanspruch in einer weltgesellschaftlichen Perspektive für pädagogisches Handeln zu verstehen ist. Mit dieser Perspektive wird Lernen in globalisierten sowie damit einhergehend indirekten sozialen Beziehungen verortet und „das Einüben in abstrakte Sozialität" (Scheunpflug, 2017, S. 169) als Herausforderung hervorgehoben. Die Komplexität des Verhältnisses zwischen Anwesenden und Nicht-Anwesenden wird somit als ein zentrales Thema einer Bildung in der Weltgesellschaft auf ein partizipatives und inklusives Bildungsverständnis bezogen, das auf das Verhältnis von Beteiligten und Nicht-Beteiligten, Eingeschlossenen und Ausgeschlossenen verweist.

Im Kontext einer globalisierten Schulrealität (Oelkers, 2011; Lang-Wojtasik, 2008) ist eine weltgesellschaftliche Perspektive umzusetzen, in der der bestehenden Heterogenität aller Beteiligten bzw. dem geführten Heterogenitätsdiskurs (dazu kritisch Budde, 2012) als Bedingung pädagogischen Handelns von Lehrkräften Rechnung getragen wird. Unterschiedliche Dimensionen sozialer Differenzsetzung werden im schulischen Kontext bedeutsam (Scheunpflug, 2011; Sturm, 2016) bzw. bedeutsam gemacht und müssen auch machtkritisch reflektiert werden (Budde, 2012; Emmerich \& Hormel, 2013). Wenning (2004) weist darauf hin, dass Heterogenität als Leitbild zu kurz greift, weil dem auch immer Homogenität gegenübersteht und dieses Spannungsverhältnis von Gleichheit und Differenz „[...] nicht einseitig aufzulösen ist“ (ebd., S. 565); eine Einschätzung, die sich auch in den Arbeiten zur Dialektik der Differenz von Edelmann (2007) zeigt. Aktuell steht jedoch zumeist der adäquate Umgang mit Heterogenität im Fokus der pädagogischen und erziehungswissenschaftlichen Debatten; eine Perspektive, die durch die politische Forderung der Inklusion noch einmal verstärkt wird. Wird dafür der Zugang zu dem Thema über die Frage nach der Professionalisierung von Lehrkräften gewählt, besteht die Gefahr, dass die Erwartung eines partizipativen und weltgesellschaftlich orientierten inklusiven Bildungsangebotes vor allem als Verantwortung der/des Einzelnen gehandhabt wird. Ungeachtet der Frage der Machbarkeit wird die Umsetzung im Alltag an Lehrkräfte weitergegeben. Bezogen auf das Thema Inklusion wurde die Forderung einer nahezu unhinterfragten Übertragung des politischen Auftrags der Inklusion in die Erziehungswissenschaft bereits kritisch kommentiert (DGfE, 2017).

Im Folgenden wird zunächst ein Überblick über (bildungs-)politische Forderungen nach Inklusion und Partizipation einerseits und Befunde aus der Forschung zu Partizipation als aktive Teilhabe in Bildungskontexten anderseits gegeben (2). Vor dem Hintergrund des Anspruchs einer partizipativen und inklusiven Bildung wird anschließend der Vorschlag gemacht, eine analytische Trennung zwischen struktureller Ebene und der Ebene von schulischen Alltagspraktiken vorzunehmen. Auf der Ebene schulischer Praktiken wird das Spannungsverhältnis von Inklusion und Exklusion sodann vor dem Hintergrund der Forderung nach Partizipation näher bestimmt und auf den Anspruch einer weltgesellschaftlichen Perspektive bezogen (3). Der Beitrag schließt mit Anregungen für die Reflexion pädagogischen Handelns im Globalen Lernen und einer Bildung für nachhaltige Entwicklung (4).

\section{Partizipation aus (bildungs)politischer und theoretischer Perspektive}

(Bildungs-)politische Perspektiven

Partizipation als aktive Teilhabe ist aus unterschiedlichen pädagogischen Denkschulen und Bildungskonzepten heraus schon lange und immer wieder als Anforderung für die pädagogische Praxis formuliert worden und rückt mit der politischen Forderung nach Inklusion erneut zentral in den Fokus. Dabei scheint eine gemeinsame Verwendung der Begriffe Inklusion - insbesondere in einem weiten Inklusionsverständnis (Ainscow, 2005) - und Partizipation in bildungspolitischen und pädagogischen Diskursen durchaus üblich zu sein. Sie ist z. B. in der englischsprachigen, völkerrechtlich verbindlichen Fassung der UN-Behindertenrechtskonvention zu finden, in welcher der Grundsatz der Partizipation als Mitbestimmung von Menschen mit Behinderung in allen gesellschaftlichen Bereichen in $\$ 3$ aufgeführt ist. Die Monitoring-Stelle zur UN-Behindertenrechtskonvention in Deutschland schreibt dazu: „Der Grundsatz der Partizipation steht mit anderen Grundsätzen, etwa dem der Inklusion und der Nichtdiskriminierung, in enger Verbindung" (Institut für Menschenrechte, 2010) und hebt die Bedeutung von Partizipation im Sinne von aktiver Mitbestimmung als einer Querschnittsaufgabe der Konvention noch einmal besonders hervor (ebd.). Auch die Europäische Kommission verbindet Inklusion und Partizipation eng miteinander: „Active inclusion means enabling every citizen, notably the most disadvantaged, to fully participate in society" (Europäische Kommission, o. J.). In einem solchen Verständnis soll Inklusion zu aktiver Teilhabe führen und wird dementsprechend als vollumfängliche Forderung an Gesellschaft und Schule gestellt. Zusätzlich wird die Forderung nach Teilhabe und Beteiligung mit dem Ziel einer nachhaltigen Bildung in Verbindung gebracht (ebd.; UN, 2015). Partizipation und Inklusion sind in entsprechendem Verständnis zunehmend auch Bestandteil entwicklungspolitischer (DBR \& VENRO, 2016) und pädagogischer Reflexionen im Rahmen einer Bildung für nachhaltige Entwicklung und des Globalen Lernens (Führing \& Böhme, 2015; Roncevic, 2018). Deutlich herausgestellt wird, dass eine Mitbestimmung aller entsprechend ihrer jeweiligen Bedürfnisse als eng mit der politischen Agenda einer inklusiven und nachhaltigen Gesellschaft verwoben zu verstehen ist. Partizipation und Inklusion sind demnach ohne einander nicht umsetzbar, definieren sich gewissermaßen gegenseitig und werden als zentraler Bestandteil einer zukunftsfähigen und global gerechten Entwicklung gesehen.

\section{Theoretische Perspektiven}

Blickt man auf theoretische Partizipationsmodelle so scheint geteilt zu sein, dass es eine Art Entwicklung hin zu Partizipationsfähigkeit aber auch in den Partizipationsmöglichkeiten braucht, es also auch Abstufungen in Partizipation und Nicht-Partizipation gibt, die zum Gegenstand kritischer Betrachtung werden müssen, wenn es am Ende um echte Partizipation gehen soll. In diesen Modellierungen wird teils zwischen sozialer und politischer Partizipation unterschieden, auch wenn das eine mit dem anderen als eng verknüpft betrachtet wird (Derecik, Goutin \& Michel, 2018, S. 28). Hart (2008) entwirft in den 1970er-Jahren die Leiter der politischen Partizipation mit einem Fokus auf junge Menschen („Ladder of 
young people's participation“). Das acht Sprossen umfassende Leitermodell bezieht auf den drei untersten Ebenen auch Formen der Zusammenarbeit ein, die als (noch) nicht partizipativ gelten: Hierzu zählt die Einbindung in Form von Manipulation, die Einbeziehung der Perspektive Jugendlicher als „dekoratives“ Element sowie eine Teilhabe nur im Rahmen eines vorab festgelegten Bewertungsschemas. Ein Stufenmodell von Partizipation findet sich bei Wright, von Unger und Block (2010) im Rahmen der Gesundheitsfürsorge („Public Health“), das die vier Bereiche Nicht-Partizipation, Prozeduren hin zu Partizipation, Partizipation sowie den Bereich der Selbst-Organisation umfasst, der über Partizipation hinausgeht. Die Darstellungsform des Stufenmodells wird auch von Schröder (1995) aufgegriffen, dessen Modell auf den schulischen Kontext angewendet werden kann (Wagener, 2013; Derecik, Goutin \& Michel, 2018). Dieses nimmt den Grad der Selbstbestimmung als Ausgangspunkt der Betrachtung von Partizipationssituationen. Blandow, Gintzel und Schröder (1999) legen ein Modell sozialer Partizipation vor und fokussieren das Maß der Entscheidungsmacht in Kommunikations- und Kooperationsprozessen. Für Straßburger und Rieger (2019) findet Partizipation nur dann statt, wenn tatsächlich Einfluss auf das Ergebnis genommen werden kann. Oser und Biedermann (2006) unterscheiden Arten von Partizipation nach ihrer Intensität, die sich unter anderem durch „die Grade der partizipativen Verantwortung, Zuständigkeit und Verpflichtung“ (ebd., S. 27) unterscheiden. Diese Unterscheidung soll helfen, sich darüber zu verständigen, was gemeint ist, wenn von Partizipation die Rede ist. Wagener (2013) stellt resümierend fest, dass „unterschiedliche Stufen der politischen und sozialen Partizipation “ nur ,in Abhängigkeit der Situation [...] ermöglicht bzw. erreicht" (ebd., S. 20) werden (können). Dies verweist im Kern darauf, dass Partizipation in ihrer konkreten Umsetzung kontextgebunden ist und entsprechend ihrer Prozesshaftigkeit nicht als starres Modell besteht.

Partizipation ist demnach als als ein (Entwicklungs-) Prozess zu verstehen, der jedoch nicht ausschließlich eindimensional linear verläuft. Dabei muss die Zielsetzung Partizipation immer auch kritisch vor dem Hintergrund einer Selektivität in Partizipationsprozessen selbst betrachtet werden, die abhängig von Zugängen, Ressourcen, Bildungshintergrund, Kompetenzen und auch Bereitschaften sind (z. B. Senatsverwaltung für Stadtentwicklung und Umwelt Berlin, 2012). Das Aufeinandertreffen unterschiedlicher Beteiligungserfahrungen verlangt somit eine kritische Auseinandersetzung mit Strukturen in partizipativ angelegten Prozessen. So zeigen Derecik, Goutin und Michel (2018) anhand der Voraussetzungen für partizipative Bildung (in der Ganztagsschule) auf, dass ein ganzes Konglomerat an Faktoren Partizipationsvorhaben beeinflussen kann.

Nicht nur die strukturellen, sondern auch die personalen Voraussetzungen stecken die Rahmenbedingungen solcher Vorhaben auf Seiten der Schülerinnen und Schüler sowie der Lehrkräfte ab. Eine Verankerung von Partizipation im Schulprogramm kann dabei eine stark unterstützende Funktion übernehmen, hängt aber immer auch in der konkreten unterrichtlichen Umsetzung von der pädagogischen Haltung der Lehrkräfte und den entsprechenden Kompetenzen der Lernenden ab (ebd., S. 53 ff.).
Im Rahmen der (bildungs-)politischen Inklusionsdebatte wird Partizipation zu Recht als umfassender normativer Anspruch formuliert. Bezogen auf die pädagogische Praxis und unter Berücksichtigung der Modellierungen von Partizipationsprozessen ergeben sich jedoch Rückfragen zur professionellen Gestaltung dieses Anspruchs. Für ein besseres Verständnis dieser aus Sicht pädagogischen Handelns formulierten Fragen nach Partizipation im pädagogischen Alltag wird im Folgenden zunächst eine theoretische Verortung des Verhältnisses von Inklusion und Exklusion vorgestellt, um im Anschluss daran einen Vorschlag für einen theoretischen Zugang zu weltgesellschaftlich ausgerichteter partizipativer Bildungsarbeit zu formulieren.

\section{Pädagogische Praktiken im Spannungsfeld von Exklusion und Inklusion Inklusion und Exklusion in der pädagogischen Praxis}

Wie bereits vielfach angemerkt, wird die (bildungs-)politische Debatte um Inklusion oftmals ohne Verweis auf Exklusion geführt. Dabei stellt das Begriffspaar Inklusion - Exklusion ein „facettenreiches konzeptionelles Instrument“ (Stichweh, 2005, S. 7) dar, dessen Verhältnisbestimmung davon abhängig ist, ob es für analytische, politische und/oder sozialkritische Zwecke Verwendung findet (Gertenbach, 2008, S. 309). Während die politische und sozialkritische Nutzung nahezu ausschließlich den Diskurs um Bildungsgerechtigkeit und den damit verbundenen Forderungen prägt, wird eine analytische Perspektive eher selten angeboten. Terhart und von Dewitz (2018) zeigen unter Rückgriff auf gesellschaftstheoretische Positionen zum Wechselverhältnis sozialer Inklusion und Exklusion am Beispiel einer Analyse zum Unterricht für neu zugewanderte Kinder und Jugendliche auf, dass die formale Einbindung in das Bildungssystem nicht automatisch zu einer Inklusion in Bezug auf Partizipationsmöglichkeiten in der sozialen Situation Unterricht führt. In der Bezugnahme auf institutionelle Strukturen einerseits (Wer nimmt wann an welchem Unterricht der Schule teil?) und den unterrichtlichen Praktiken (Was passiert im Unterricht?) andererseits, werden Dynamiken inkludierender Exklusion und exkludierender Inklusion deutlich, die Bourdieu et al. in der Figur des „intern Ausgeschlossenen“ (1998, S. 527) verdichten. Daran wird deutlich, dass die Verknüpfung der strukturellen und schulischen Ebene und dem, was im Unterricht stattfindet, für das Verständnis von Partizipation von zentraler Bedeutung ist.

Das damit angesprochene Verhältnis von Struktur und Praktiken zeigt sich auch bei Sturm (2016), die bezogen auf eine Inklusive Bildung zwischen der Ebene des programmatischen Rahmenkonzepts Inklusion einerseits und der Ebene der darin eingebetteten schulischen Praxis andererseits unterscheidet (Sturm, 2016, S. 133 ff.). Hat das Rahmenkonzept der Inklusion die möglichst umfassende Teilhabe aller zum Ziel, werden auf der Ebene der schulischen Praktiken immer auch exkludierende Erfahrungen gemacht, die - wenn sie kommunikativ bearbeitet werden können - nicht an sich marginalisierend wirken müssen. Die von Sturm angeführte Unterscheidung zeigt auf, dass im Kontext eines normativen inklusiven Rahmenkonzeptes, die darin eingebetteten Praktiken als „kon- 
tinuierliches Wechselspiel von Inklusion und Exklusion aus konkreten unterrichtlichen und schulischen Interaktionsbezügen" (Sturm, 2016, S. 139) zu verstehen sind, das sich durch ein "gegenseitige[s] Lernen voneinander und übereinander auszeichnet" (ebd.). In einem inklusiven Rahmenkonzept sind diese „Momente von Zugehörigkeit und Nichtzugehörigkeit“ (ebd.) situativ und nicht systematisch zu verstehen. Das Bestehen eines solchen inklusiven Grundverständnisses sowie der situativen Bearbeitung von Differenzerfahrungen ist zugleich institutionelle Aufgabe und Anspruch an das Handeln von Lehrkräften. Bezogen auf das Verhältnis von Struktur und Praxis schreibt Sturm (2016): „Eine veränderte Bearbeitung von Heterogenität ist weder ausschließlich bei Lehrkräften zu verorten, d. h. sie kann nicht von ihnen allein verantwortet und/ oder überwunden werden, noch durch ausschließliche Veränderungen der formalen Regeln der Organisation Schule. Die unterrichtlichen und schulischen Regeln und Praktiken sind in gesellschaftliche Kräfte- und Machtverhältnisse“ (ebd., S. 131) eingebunden.

\section{Partizipationsprozesse unter Berücksichtigung abstrakter Sozialität}

Die Unterscheidung zwischen Struktur und Praktiken wird im Folgenden als Heuristik zur Verhältnisbestimmung zwischen normativem Anspruch und deskriptiv-analytischer Verwendung für ein partizipativ angelegtes Verständnis Globalen Lernens genutzt. Dabei wird deutlich, dass sich auf der Ebene der Praktiken das Verhältnis von Inklusion und Exklusion im Kontext Globalen Lernens noch einmal in besonderer Weise akzentuiert. In einer weltgesellschaftlichen Perspektive auf Schule und Unterricht wird die Komplexität durch die zu berücksichtigenden Akteurinnen und Akteure und das Einüben in eine „abstrakte Sozialität“, die durch globale und lokale Kräfte- und Machtverhältnisse durchdrungen ist, sehr deutlich und explizit zum Gegenstand. Wie also kann das gegenseitige von- und übereinander Lernen in weltgesellschaftlichem Bezug stattfinden? Für das Handeln von Lehrkräften erhält die Berücksichtigung Abwesender sowohl im Sinne der inhaltlichen Erarbeitung unterschiedlicher Perspektiven und Interessenlagen, als auch bei der methodisch-didaktischen Gestaltung im Unterricht, z. B. in Form des Austauschs mit internationalen Akteurinnen und Akteure, Bedeutung. Das Potenzial von Ein- und Ausschluss, vor allem auch aufgrund notwendiger Komplexitätsreduktionen für den Unterricht, steigt durch die Aufhebung einer nationalen Engführung noch einmal exponentiell an.

Auch die von Sturm geforderte Verhandlung und Auseinandersetzung mit Differenzerfahrungen erfährt im Rahmen des Globalen Lernens und einer Bildung für nachhaltige Entwicklung eine spezifische Ausrichtung. Dies wird etwa an dem Verhältnis von Nord und Süd und den damit verbundenen Differenzen deutlich, denen im Globalen Lernen eine zentrale Bedeutung zukommt. Als analytische Unterscheidung bildet diese Kategorisierung einerseits die Grundlage, um damit verknüpfte Ungleichheitsverhältnisse sichtbar zu machen; anderseits muss die Unterscheidung zwischen globalem Süden und Norden selbst zugleich zum Gegenstand von Reflexionen gemacht werden, da sie nicht nur abstrakte, sondern ebenso direkt in der Situation des Unterrichts bedeutsame Adressierungen und möglichen Ein- oder Ausschluss von Personen oder
Merkmalen bewirken kann. Nicht zuletzt ist die Frage der Gerechtigkeit, die im Zentrum des normativen Anspruchs von Inklusion und Partizipation steht, wiederum Kernfrage des Globalen Lernens selbst und muss zum Gegenstand unterrichtlicher Interaktion werden. Diese Thematisierung kann eine reflexive Bearbeitung des unterrichtlichen Geschehens bezogen auf Inklusion und Partizipation bewirken, was eine besondere Sensibilität der Lehrkraft verlangt.

Die gewählte Heuristik trägt dazu bei, eine weltgesellschaftliche Perspektive in der engen Verzahnung von strukturellen und institutionellen Rahmenbedingungen einerseits und der schulischen Praktiken, die zentral durch das Handeln von Lehrkräften mitbestimmt werden, andererseits, einzuordnen. Lehrkräfte nehmen dabei „eine Schlüsselstellung ein, da ihr explizit-kommunikativer Umgang mit Differenz [und Gemeinsamkeiten, die Autorinnen] im Unterricht ebenso wie ihre Handlungspraxis, den Rahmen der Differenzbearbeitung durch die Schüler/innen sowie deren Lern- und Bildungsmöglichkeiten entscheidend prägen" (Florian und Rouse, 2010, S. 185 zit. nach Sturm, 2016, S. 132). Damit strukturieren Lehrkräfte Partizipationsangebote entscheidend mit, indem sie auf der Mikroebene für die Ausgestaltung ihres Handlungsspielraumes verantwortlich sind (Miller, 2013). Im Folgenden werden Ansatzmöglichkeiten für die Reflexion und Weiterentwicklung eines partizipativ ausgerichteten Globalen Lernens und einer Bildung für nachhaltige Entwicklung aufgezeigt.

\section{Implikation für das pädagogische Handeln von Lehrkräften im Globalen Lernen - ein Ausblick}

In dem Beitrag wurde einleitend die Forderung nach Partizipation als Möglichkeit der aktiven Mitbestimmung und des Gestaltungsauftrags für das Globale Lernen und eine Bildung für nachhaltige Entwicklung benannt. Für ein besseres Verständnis der Forderung nach aktiver Teilhabe im Feld von Bildung wurde anschließend auf die zumeist enge Verzahnung von Partizipation und einem inklusiven Bildungsverständnis verwiesen sowie auf Basis theoretischer Modellierungen ein prozesshaftes, zumeist nichtlineares Verständnis von Partizipation entwickelt. Vor dem Hintergrund der (bildungs-)politischen Inklusionsdebatte wurde die professionelle Gestaltung einer partizipativen pädagogischen Praxis in den Blick genommen. Mit der analytischen Unterscheidung zwischen Struktur und Praktiken wurde für ein differenziertes Verständnis von Inklusion und Exklusion plädiert und eine partizipativ angelegte weltgesellschaftliche Perspektive entwickelt. Normative konzeptionelle Anforderungen eines inklusiven, partizipativen und nachhaltigen Verständnisses von Bildung können damit analytisch von der praktischen Umsetzung unterschieden werden, die situativ zwischen Teilhabe und Nichtteilhabe wechselt. Als theoretische Trennung kann der Partizipationsanspruch im Globalen Lernen sowie einer Bildung für nachhaltige Entwicklung auf diese Weise für die Praxis anschlussfähig gemacht werden. Die analytische Trennung ist explizit nicht dazu gedacht, exkludierende Praktiken sowie die systematische Verweigerung aktiver Teilhabe zu rechtfertigen. Vielmehr lassen sich aus diesen theoretischen Überlegungen heraus Anregungen für die Praxis und Theorieentwicklung des Globalen Lernens ableiten. 
Bezogen auf den partizipativen Anspruch erscheint der in den Modellen idealtypisch dargestellte schrittweise Aufbau von wenig hin zu viel Beteiligung (bis hin zu Partizipation übersteigende selbstbestimmte Formen) in der Praxis weniger eindeutig und muss als ein beständiges Aushandeln im Alltag reflektiert werden, was die strukturell legitime Forderung nach mehr Partizipation anstrebt. Vor dem Hintergrund der alltäglichen Aufgabe im Unterricht situativ zwischen den Möglichkeiten der Beteiligung und der Nicht-Beteiligung unter Berücksichtigungbestehender Differenzund weltgesellschaftlicher Realität zu entscheiden, ist anspruchsvoll. Diese Entscheidungen treffen Lehrkräfte tagtäglich vor dem Hintergrund von Motiven, die von diagnostischem Wissen und methodischdidaktischen wie auch inhaltlichen Überlegungen bestimmt werden. Zentral dabei ist für das Handeln der Lehrkräfte, soziale Differenzsetzungen nicht als absolut und systematisch zu verstehen. Entscheidend für die Ausgestaltung der Situationen ist, dass die Erfahrungen des Nicht-Mitmachens und der Exklusion bearbeitbar und flexibel bzw. zeitlich begrenzt sind. In der Schule ist es daher bedeutsam, dass die konkrete Erfahrung, mit anderen etwas zu teilen und in anderen Dingen verschieden zu sein, nicht als unbefragte Tatsache vorausgesetzt, sondern selbst (und auch in ihrer sozialen Konstruiertheit) zum Thema gemacht wird und dies in einem wertschätzenden pädagogischen Rahmen (vgl. auch Sturm, 2016).

Die weltgesellschaftliche Tatsache und die Bearbeitung global gesellschaftlicher Fragestellungen akzentuiert diese pädagogische Aufgabe, indem Nicht-Anwesende mitgedacht werden sollen. Die Anlage dieses Mitdenkens und wie Weltgesellschaft situativ repräsentiert wird, unterliegt aber ebenso pädagogisch-didaktischen Entscheidungen, die Ein- und Ausschluss bedingen. Nicht in jeder Situation kann über alle Perspektiven auf ein Thema, können alle Akteurinnen und Akteure zu einem Thema berücksichtigt werden. Auf der Ebene der schulischen Praktiken müssen demnach vor allem Reflexionsanlässe in der Lehrkräfteaus- und -fortbildung geschaffen werden, z. B. im Sinne einer Anregung zu einer dezidierten Reflexion eigener Differenzkonstruktionen, von Strategien der Gruppenbildung, der Ansprache und rhetorischen Mustern in der Interaktion, Bewertungsmaßstäben oder Öffnungsmöglichkeiten von Unterricht hin zu einer Schüler/-innenbeteiligung und Empowerment, wie es auch in anderen pädagogischen Ansätzen angeregt wird, die sich in diskriminierungskritischer Absicht mit Differenzsetzungen befassen (exempl. Leiprecht, 2015; 2018). Die Verbindung der analytischen Begriffspaare Struktur und Praktiken sowie Inklusion und Exklusion kann zu einer theoretischen Ausdifferenzierung in der Erarbeitung von Handlungsstrategien für den Umgang mit unterschiedlichen Akteure und Akteurinnen beitragen. Die Selbstreflexion eigener Bildungsansprüche wird so zum Thema gemacht, was auf die Potenziale einer stärkeren Verbindung zwischen erziehungswissenschaftlichen, allgemein didaktischen und dem globalen Lernen bzw. einer Bildung für nachhaltige Entwicklung neuerlich hinweist.

In der analytischen Verwendung von Exklusion und Inklusion zur Betrachtung der pädagogischen Praxis und der heuristischen Unterscheidung von Struktur und Praktik wird möglicherweise die häufig formulierte Verunsicherung und Überforderung im Rahmen von Umsetzungsbemühungen
Globalen Lernens bzw. einer Bildung für nachhaltige Entwicklung (Brock \& Grund, o. J.) und Inklusion (Braun, 2014) greifbar. Vor dem Hintergrund eines partizipativen und inklusiven Anspruchs der Einbindung aller wird sichtbar, dass es auf der Ebene des (unterrichtlichen) Handelns zu situativ gestalteter Teilhabe und nicht Teilhabe kommt, die durch Lehrkräfte (aber auch durch Schüler/inne/n untereinander) vollzogen wird. Partizipativ handeln (lernen) heißt somit, die bestehenden Unterschiedlichkeiten nicht nur zu akzeptieren, sondern aktiv zum Thema in Entscheidungsprozessen zu machen. Ein partizipatives Verständnis Globalen Lernens im Rahmen des inklusiven schulischen Alltags kann dazu beitragen, im Zuge des zunehmend individualisierten Blicks auf Schüler/innen das Moment der gegenseitigen Auseinandersetzung zu stärken, wobei aber auch mit diesem Anspruch zwischen den Forderungen auf normativ-politischer Ebene und der deskriptiv-analytischen Betrachtung konkreten Handelns zu unterscheiden ist.

Es wurde argumentiert, dass Globales Lernen als partizipatives Handeln auf der Ebene von Alltagspraktiken in einem Wechselspiel situativer Teilhabe und Nichtteilhabe stattfindet und dort nur in einer Balancierung und reflexiven Bearbeitung des Spannungsverhältnisses besteht. Durch eine weltgesellschaftliche Rahmung und die damit einhergehende Aufgabe, Themen sozialen Zusammenlebens als globale Fragestellungen zu bearbeiten, wird die Komplexität von Beteiligung und Nicht-Beteiligung und dem Ein- und Ausschluss von zu berücksichtigenden Akteuren und Akteurinnen noch einmal erhöht. Gleichwohl kann eine weltgesellschaftliche Perspektive dazu beitragen, die oftmals auf nationalstaatliche Kontexte reduzierte Frage von Partizipation in pädagogischen Kontexten zu bereichern und die Gestaltung gesellschaftlicher Entwicklung in globaler Perspektive stärker in den Blick zu nehmen. Mit der Unterscheidung zwischen programmatischem Rahmenkonzept und der Deskription pädagogischer Praktiken soll ein Beitrag geleistet werden, Verunsicherung und Überforderung derjenigen, die für die Umsetzung von Inklusion, Globalem Lernen und dem damit verbundenen Anspruch der Partizipation verantwortlich zeichnen, einzuordnen. Die gewählten theoretischen Differenzierungen tragen aus unserer Sicht dazu bei, Akteurinnen und Akteure in der Reflexion der eigenen Ansprüche zwischen den Anforderungen eines normativen Rahmenkonzepts einerseits und den alltäglichen Handlungsspielräumen einer auf die Teilhabe aller hin ausgerichteten Bildung andererseits zu unterstützen. Nicht zuletzt eröffnet das Globale Lernen für den Diskurs um Inklusion eine weltgesellschaftliche Rahmung des geteilten partizipativen Anspruchs.

\section{Literatur}

Aincow, M. (2005). Developing inclusive education systems: what are the levers for change? Journal of Educational Change, 6, 109-124. https://doi.org/10.1007/s1083 3-005-1298-4

Blandow, J., Gintzel, U., \& Hansbauer, P. (1999). Partizipation als Qualitätsmerkmal in der Heimerziehung: eine Diskussionsgrundlage. Münster: Votum.

Böhme, L. (2019). Politische Bildung für Schülerinnen und Schüler mit sonderpädagogischem Förderbedarf: Perspektiven Globalen Lernens an Förderzentren. Frankfurt am Main: Wochenschau-Verlag.

Bourdieu, P. et al. (1997). Das Elend der Welt. Zeugnisse und Diagnosen alltäglichen Leidens an der Gesellschaft. Konstanz: Universitäts-Verlag Konstanz. 
Braun, N. (2014). Überfordert die Inklusion Lehrer? Immer mehr geraten unter Druck. Grundschule, (5), 30-31.

Brock, A. \& Grund, J. (Wissenschaftliche Beratung Weltaktionsprogramm BNE) (o. J.). Executive Summary. Bildung für eine nachhaltige Entwicklung in Lehr-Lernsettings: Quantitative Studie des nationalen Monitorings - Befragung von LehrerInnen. Zugriff am 11.10.2019 https://www.bne-portal.de/sites/default/files/downloads/ Nationales\%20Monitoring_Quantitative\%20Studie_LehrerInnen.pdf

Budde, J. (2012). Problematisierende Perspektiven auf Heterogenität als ambivalentes Thema der Schul- und Unterrichtsforschung. Zeitschrift für Pädagogik, 58(4), 522-540.

Budde, J. (2017). Heterogenität: Entstehung, Begriff, Abgrenzung. In T. Bohl, J. Budde \& M. Rieger-Ladich (Hrsg.), Umgang mit Heterogenität in Schule und Unterricht (S. 13-26). Bad Heilbrunn: Klinkhardt (utb).

Bundesministerium für wirtschaftliche Zusammenarbeit und Entwicklung (BMZ) \& Kultusministerkonferenz (KMK). (2016). Orientierungsrahmen für den Lernbereich Globale Entwicklung im Rahmen einer Bildung für nachhaltige Entwicklung. Bonn, Berlin.

De Haan, G. (2010). Bildung für nachhaltige Entwicklung: Ein neues Lern- und Handlungsfeld. Herausforderung - Kernthemen - Strategien. Lernende Schule, 13(50), 6-9.

Derecik, A., Goutin, M.-C. \& Michel, J. (2018). Partizipationsförderung in Ganztagsschulen: Innovative Theorien und komplexe Praxishinweise. Wiesbaden: Springer Fachmedien Wiesbaden. https://doi.org/10.1007/978-3-658-17072-1

Deutscher Behindertenrat \& Verband Entwicklungspolitik Deutscher Nichtregierungsorganisationen e. V. (2016). Die Umsetzung der globalen 2030-Agenda für nachhaltige Entwicklung inklusiv gestalten: Forderungspapier. Zugriff am 19.09.2019 https://venro.org/fileadmin/user_upload/Dateien/Daten/Publikationen/Stellung nahmen/VENRO-DBR_Forderungspapier_Nachhaltigkeitsstrategie.pdf

DGfE - Deutsche Gesellschaft für Erziehungswissenschaft (2017). Stellungnahme der Deutschen Gesellschaft für Erziehungswissenschaft: Inklusion. Zugriff am 07.09.2019 https://www.dgfe.de/fileadmin/OrdnerRedakteure/Stellungnah men/2017.01_In klusion_Stellungnahme.pdf

Edelmann, D. (2007). Pädagogische Professionalität im transnationalen sozialen Raum. Eine qualitative Untersuchung über den Umgang von Lehrpersonen mit der migrationsbedingten Heterogenität ihrer Klassen. Wien, Zürich: LIT.

Emmerich, M. \& Hormel, U. (2013). Heterogenität-Diversity-Intersektionalität: Zur Logik sozialer Unterscheidungen in pädagogischen Semantiken der Differenz. Wiesbaden: Springer VS. https://doi.org/10.1007/978-3-531-94209-4

Erdsiek-Rave, U. \& John-Ohnesorg, M. (2015). Demokratie lernen - eine Aufgabe der Schule? 1. Auflage. Schriftenreihe des Netzwerk Bildung [35]. Berlin: Friedrich-Ebert-Stiftung. Zugriff am 25.09.2019 http://library.fes.de/pdf-files/studien foerderung/12035.pdf

Europäische Kommission (o. J.). Active inclusion. Zugriff am 07.09.2019 https:// ec.europa.eu $/$ social $/$ main.jsp?catId=1059\&langId=en

Florian, L. \& Rouse, M. (2010). Teachers' professional learning and inclusive practice. In R. Rose (Hrsg.), Confronting obstacles to inclusion: international responses to developing inclusive schools (S. 185-199). Abington, United Kingdom: Routledge.

Führing, G. \& Böhme, L. (2015). Globales Lernen inklusiv?! Theoretische und praxisrelevante Überlegungen. Berlin: BGZ.

Gertenbach, L. (2008). Ein „Denken des Außen“- Michel Foucault und die Soziologie der Exklusion. Soziale Systeme, 14(2), 308-328. https://doi.org/10.1515/sosy s-2008-0211

Hart, R. (2008). Stepping Back from 'The Ladder': Reflections on a Model of Participatory Work with Children. In A. Reid, B. B. Jensen, J. Nikel \& V. Simovska (Hrsg.), Participation and Learning. Perspectives on Education and the Environment, Health and Sustainability (S. 19-31). Springer Verlag. https://doi.org/10.1007/9781-4020-6416-6_2

Institut für Menschenrechte (2010). Partizipation - ein Querschnittsanliegen der UN-Behindertenrechtskonvention. Zugriff am 07.09.2019 https://www.institutfuer-menschenrechte.de/uploads/tx_commerce/Positionen_nr_3_Partizipation_ ein_Querschnittsanliegen_der_UN_Behindertenrechtskonvention.pdf

Lang-Wojtasik, G. (2008). Schule in der Weltgesellschaft. Herausforderungen und Perspektiven einer Schultheorie jenseits der Moderne. Weinheim und München: Juventa. Leiprecht, R. (2015). Zum Umgang mit Rassismen in Schule und Unterricht. In R. Leiprecht \& A. Steinbach (Hrsg.), Schule in der Migrationsgesellschaft (S. 115-149). Schwalbach i. T.: Wochenschau.

Leiprecht, R. (2018). Rassismuskritische Ansätze in der Bildungsarbeit. In I. Gogolin, V. Georgi, M. Krüger-Potratz, D. Legyel \& U. Sandfuchs (Hrsg.), Handbuch Interkulturelle Pädagogik (S. 255-260.). Stuttgart: UTB.

Miller, S. (2013). Die Sicht der Lehrkräfte auf Heterogenität: Ergebnisse einer quantitativen Erhebung in NRW. In E. Jürgens \& S. Miller (Hrsg.), Ungleichheit in der Gesellschaft und Ungleichheit in der Schule: Eine interdisziplinäre Sicht auf Inklusionsund Exklusionsprozesse (S. 235-251). Weinheim: Beltz Juventa.
Oelkers, J. (2011). Globalisierung als Herausforderung für den Unterricht - Kompetenzentwicklung und Heterogenität. In W. Sander \& A. Scheunpflug (Hrsg.), Politische Bildung in der Weltgesellschaft: Herausforderungen, Positionen, Kontroversen (S. 167-189). Bonn: Bundeszentrale für politische Bildung.

Oser, F. \& Biedermann, H. (2006). Partizipation - ein Begriff, der ein Meister der Verwirrung ist. In C. Quesel \& F. Oser (Hrsg.), Die Mühen der Freiheit: Probleme und Chancen der Partizipation von Kindern und Jugendlichen (S. 17-37). Zürich Chur: Rüegger Verlag.

Reitz, S. (2015). Kinder und Jugendliche haben ein Recht auf Partizipation: Was aus menschenrechtlicher Sicht im Bildungsbereich getan werden muss. Berlin: Deutsches Institut für Menschenrechte.

Roncevic, K. (2018). Bildung für nachhaltige Entwicklung und Inklusion. Weitblick, 1 (2). Zugriff am 11.10.2019 https://germanwatch.org/en/node/15530

Scheunpflug, A. (2011). Lehren angesichts der Entwicklung zur Weltgesellschaft. In W. Sander \& A. Scheunpflug (Hrsg.), Politische Bildung in der Weltgesellschaft: Herausforderungen, Positionen, Kontroversen (S. 204-215). Bonn: Bundeszentrale für politische Bildung.

Scheunpflug, A. (2017). Globales Lernen - Theorie. In G. Lang-Wojtasik \& U. Klemm (Hrsg.), Handlexikon. Globales Lernen. Ulm: Klemm+Oelschläger.

Schröder, R. (1995). Kinder reden mit! Beteiligung an Politik, Stadtplanung und -gestaltung. Weinheim: Beltz.

Senatsverwaltung für Stadtentwicklung und Umwelt Berlin (2012). Handbuch zur Partiziptation. Berlin.

Stichweh, R. (2005). Inklusion und Exklusion. Studien zur Gesellschaftstheorie. Bielefeld: transcript.

Stoltenberg, U. (2007). Partizipation und Nachhaltigkeit. Vom Leitbild zur Umsetzung. In H. Jonuschat, E. Baranek, M. Behrendt, K. Dietz, B. Schlußmeier, H. Walk, \& A. Zehm (Hrsg.), Ergebnisse sozial-ökologischer Forschung: Vol. 7. Partizipation und Nachhaltigkeit: Vom Leitbild zur Umsetzung [Ergebnisse des Workshops „Partizipation und Nachhaltigkeit - Der Teufel steckt im Detail“, der am 23. September 2005 in Berlin stattfand (S. 54-66). München: Oekom.

Straßburger, G. \& Rieger, J. (2019). Partizipation kompakt: Für Studium, Lehre und Praxis sozialer Berufe. Weinheim, Basel: Beltz Juventa.

Sturm, T. (2016). Lehrbuch Heterogenität in der Schule (2. Auflage). München: Reinhardt Verlag.

Terhart, H. \& Dewitz, N. v. (2017). Newly arrived migrant students in German schools: Exclusive and inclusive structures and practices. European Educational Research Journal, 17(2), 290-304. https://doi.org/10.1177/1474904117722623

UN (2015). Transforming our world: the agenda 2030 for sustainable development. Zugriff am 24.09.2019 https://sustainabledevelopment.un.org/post2015/trans formingourworld/publication.

Wagener, A. L. (2013). Partizipation von Kindern an (Ganztags-)Grundschulen. Ziele, Möglichkeiten und Bedingungen aus Sicht verschiedener Akteure. Weinheim: Beltz Verlag.

Wenning, N. (2004). Heterogenität als neue Leitidee der Erziehungswissenschaft? Zur Berücksichtigung von Gleichheit und Verschiedenheit. Zeitschrift für Pädagogik, 50(4), 565-582.

Widmaier, B. \& Steffens, G. (Hrsg.) (2010). Weltbürgertum - Kosmopolitisierung - politische Bildung. Schwalbach.

Wright, M.T., von Unger, H. \& Block, M. (2010). Partizipation der Zielgruppe in der Gesundheitsforschung und Prävention. In M.T. Wright (Hrsg.), Partizipative Qualitätsentwicklung in der Gesundheitsförderung und Prävention (S. 35-52). Bern: Verlag Hans Huber.

\section{Dr. Lydia Kater-Wettstädt}

ist Verwaltungsprofessorin für Trans- und Interkulturelle Bildung und Sachunterricht am Institut für Integrative Studien der Leuphana Universität Lüneburg. Ihre Forschungsinteressen liegen im Bereich Bildung für nachhaltige Entwicklung, Globales Lernen und deren Umsetzung und Herausforderung in der Lehrerbildung, damit verbunden z. B. die Frage nach Inklusion und Bildung für nachhaltige Entwicklung und die Rolle reflexiver Praxis.

\section{Dr. Henrike Terhart}

ist Vertretungsprofessorin für Erziehungswissenschaft mit dem Schwerpunkt Erziehung und Migration am Fachbereich Erziehungswissenschaften der Johann Wolfgang Goethe-Universität Frankfurt. Ihre Forschungsschwerpunkte sind theoretische und methodische Fragen zu Bildung und Migration, Organisationsentwicklung in der Migrationsgesellschaft und Professionalisierung in transnationaler Perspektive. 